\title{
Investigation of effects of clay content on $F-\Phi$ relationship by Lattice gas automation using digital rock model
}

\author{
Yue Wenzheng ${ }^{1,2,3 *}$, Tao Guo ${ }^{1,2,3}$, Chai Xiyuan ${ }^{4}$, Jiang Hongxiu ${ }^{4}$ and \\ Mu Hongwu ${ }^{4}$ \\ ${ }^{1}$ State Key Laboratory of Petroleum Resource and Prospecting, China University of Petroleum, Beijing 102249, China \\ ${ }^{2}$ Key Laboratory of Earth Prospecting and Information Technology, China University of Petroleum, Beijing 102249, China \\ ${ }^{3}$ CNPC Key Laboratory of Well Logging, China University of Petroleum, Beijing 102249, China \\ ${ }^{4}$ Bohai Drilling Engineering Company LTD Logging Company, Tianjin 300280, China
}

(C) China University of Petroleum (Beijing) and Springer-Verlag Berlin Heidelberg 2011

\begin{abstract}
The Lattice Gas Automation (LGA) method, which is improved by introducing a reflection coefficient for the border between phases to show its effect on current path, is used in this paper to simulate the current flow in digital rock for investigating the effects of clay content and clay distribution types on the relationship between formation factor $(F)$ and rock porosity $(\phi)$. The digital rock model is constructed by simulating a natural deposit of matrix particles with different shapes and radius. Based on the simulation results, it was found that both dispersed clay and laminated clay can lead to a non-Archie relationship of $F-\phi$. The non-Archie effect of laminated clay on the $F-\phi$ relationship was more significant than that of dispersed clay. Moreover, a realistic model is developed in this work for quantitatively describing the effect of clay content (Denoted as $V_{\mathrm{sh}}$ ) on parameters a and $\mathrm{m}$. These study results have further demonstrated the validity of LGA in study of electrical transport properties at a pore scale.
\end{abstract}

Key words: Formation factor, non-Archie, Lattice gas automation, porosity, clay content, clay distribution

\section{Introduction}

The relationship between the formation factor and rock porosity $(F-\phi)$ is essential in reservoir evaluation. Archie (1942) first proposed a quantitative formula to relate the formation factor $(F)$ to the porosity $(\phi)$ as

$$
\frac{a}{\varphi^{m}}=\frac{R_{o}}{R_{w}}=F
$$

where $R_{\mathrm{o}}$ is the resistivity of the porous rock fully saturated with water; $R_{\mathrm{w}}$ is the resistivity of the filling water in rock pores; $\phi$ is the porosity; $a$ is a real constant; $m$ is usually called the cementation factor; $F$ is the formation factor. Equation (1) indicates the $F-\phi$ relationship will be linear in log-log scale as shown in Fig. 1.

Many experiments have proved that Archie's $F-\phi$ relationship is for the pure rock without effect of clay content (Archie, 1942; Yong, 1982; Diederix, 1982; Wong et al, 1984; Herrick and Kennedy, 1994; Worthington, 2006). With the effect of clay, the $F-\phi$ relationship is often non-linear in log-

*Corresponding author. email: yuejack1@sina.com Received October 8, 2010

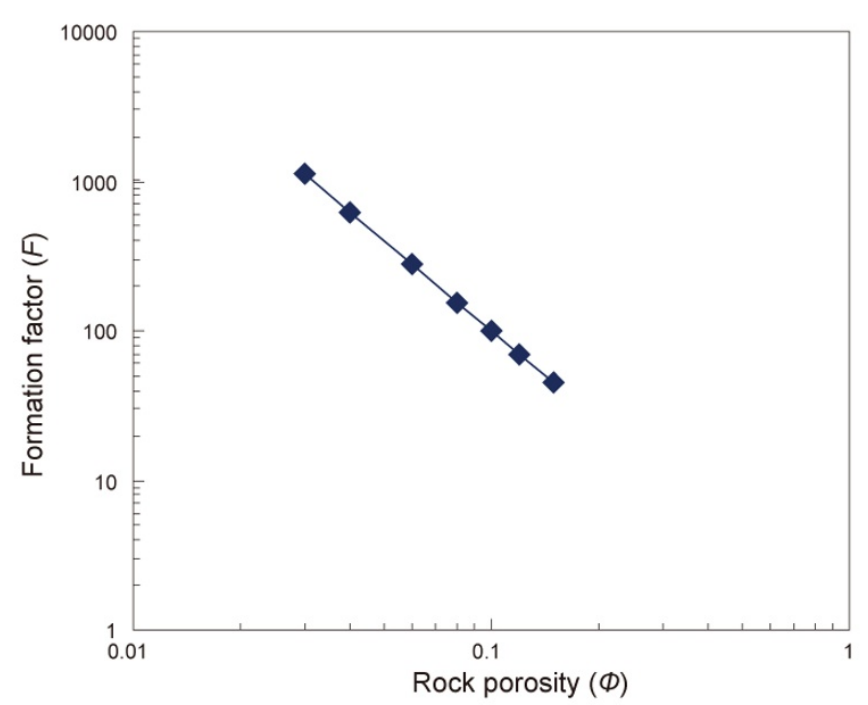

Fig. 1 F- $\phi$ relationship in Archie's equation

$\log$ scale. This is the so called non-Archie $F-\phi$ relationship (Li et al, 2008). Although a lot of experiments have been conducted for studying the non-Archie $F-\phi$ relationship, the mechanisms of clay content influencing the $F-\phi$ relationship 
is still not clear and a valid model for quantifying the effects of $V_{\text {sh }}$ on parameters $a$ and $m$ has not yet been developed. These problems can not be solved by physical rock experiments alone because the contents and microstructure of the clay minerals contained in the rock can not be measured exactly nor controlled directly during the experiments. Hence it is impossible to investigate the detailed effect of the two factors on the parameters $a$ and $m$ by changing them in a real rock experiment. Therefore, a numerical method like Lattice Gas Automation (LGA) is used in our work to study these problems due to its flexibility in changing the clay content and the clay distribution types.

The LGA method is increasingly popular in many fields especially in the micro-flow of fluid and electric current in porous medium (Li et al, 1994). In 1988, Rothman (1988) proved that the LGA method allows accurate calculations of fluids flow at the microscopic scale in grossly irregular geometries. Since 2000, the LGA (Küntz et al, 2000; Yue et al, 2005) has been applied to simulate electric current flow in a porous medium fully saturated with water, and study the relationship of formation factor and porosity. Moreover, the LGA has a wide application in geophysics ( $\mathrm{Li}$ and $\mathrm{Hu}, 1995$; $\mathrm{Hu}$ and Li, 1997; Liu et al, 1999; Wang and Li, 1999).

\section{Lattice gas automation method}

The LGA method is an evolution of Cellular Automation in updating rules and discrete space. The first model by the LGA method was developed in 1976 named HPP (Hardy, 1973), which is not accepted as a generally useful method due to its lack of rotational symmetry. In 1986, the FHP model (Frisch et al, 1986) overcame the drawbacks of HPP with a triangular lattice instead of the rectangular one and retrieved the Navier-Stokes (NS) equation at the macro-scale from collisions of the LGA particle at the micro-scale ( $\mathrm{Li}$ et al, 1994). Later, an improvement of FHP by introducing still particles makes the LGA method more reasonable for simulation of fluid flow.

The black dot in Fig. 2 represents the lattice gas particle moving along the direction of the arrow. Frisch et al (1986) discovered, by detailed derivation of the NS equation at the macro-scale from the evolution of particle collisions at the micro-scale, that the kinetic viscosity of fluids in the LGA simulation is generally dependent on the applied collision rules and average particle density. They divided the collision rules into five collision sets (from FHP1 to FHP5) in view of the viscosity to simulate five fluids.

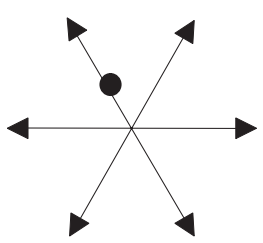

Fig. 2 Structure of discrete space for FHP model

The model used in this paper for simulation of current flow is the FHP with still particles, and the FHP2 collision is taken as a basic evolution rule of the LGA method to demonstrate the theory for simulation of current flow in porous medium. According to the approach used by Frisch et al (1986), the NS equation can be retrieved as follows with the definition of density and momentum

$$
\text { Density: } \rho=\sum_{\alpha} f_{\alpha}(\vec{x}, t)
$$

Momentum: $\rho \vec{u}=\sum_{\alpha} \vec{e}_{\alpha} \cdot f_{\alpha}$

where $f_{\alpha}(\vec{x}, t)$ is the distribution function of particles at site $\vec{x}$ and time $t ; \vec{e}_{\alpha}$ is the set of vector of unit length pointing from lattice site $\vec{x}$ to its neighbouring sites.

For an incompressible fluid with a viscosity $(\mu)$, if the velocity is sufficiently low so as to the inertial term can be ignored, the NS equation can be reduced to be

$$
V=-\frac{K}{\mu} \nabla p
$$

The expression of current density and conductivity can be derived from Maxwell's equations and Stokes's law as follows

$$
J=-\frac{T}{\mu} \nabla \psi
$$

where $T=n q^{2} / 6 \pi r, n$ is the particle density, $q$ the electric charge, $r$ the radius of particle; $\mu$ is the viscosity, $\psi$ the electric potential, $J$ the current density. Equation (4) is Darcy's law and equation (5) is Ohm's law.

By comparing the equations (4) and (5), it can be found that the two equations are the same expression if the electric charges replace the fluid particles and the electric potential replace the pressure. The similarity between Darcy's and Ohm's laws demonstrates that it is reasonable to simulate the electric current flow in porous medium with the LGA method.

\section{Digital rock examples}

The digital rock approach plays an increasingly important role in petrophysics because of its flexibility and convenience in handling complex pore geometry. It has been proved to be an effective approach to study the transport properties of porous rocks at meso-scale as a complement to physical rock experiments. The numerical algorithms for constructing a digital rock by the distribution of uniform particles and the pileup of random-sized particles have been used to investigate the physical properties of reservoir rocks. Recently, an improved algorithm has been used for digital rock modelling. In this algorithm the size of matrix grains is controlled by the porosity and size distribution obtained from laboratory experiments on real rock (Yue et al, 2009). The advantage of the improved method over the conventional method is that the distribution of particle size can be controlled to be identical with that of the real rock samples. In this paper, the digital rock examples used in the simulation are constructed by randomly pilling particles to form a pile of matrix grains with the developed algorithm (Yue et al, 2009).

The porosity and the size distribution of particles should be measured first before the construction of a digital rock. 
Images of casing thin section(CTS) of rock are often used to analyze the pore structure instead of real rock samples in consideration of convenience and low cost. An algorithm is developed in this paper to extract the information about the size distribution of matrix grains from the CTS images of rocks. Fig. 3 shows the size distribution of matrix grains extracted from a CTS image. In this figure, Fig. 3(a) shows the original CTS image and Fig. 3(b) shows the obtained radius distribution of matrix grains. Consequently, the size distribution of matrix grains can be used to construct the digital rock model through piling up the grains and compacting them into the specified porous space to investigate the electrical properties of a rock with the LGA simulation. During the compaction, the grains may be moved from their original positions to new sites for reaching the balance of contacted particles under pressure.

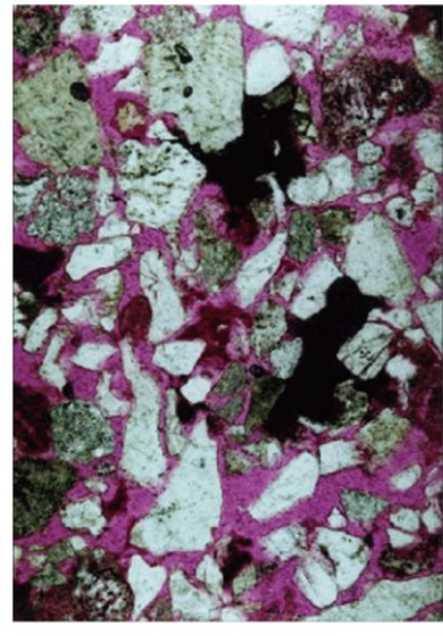

(a)

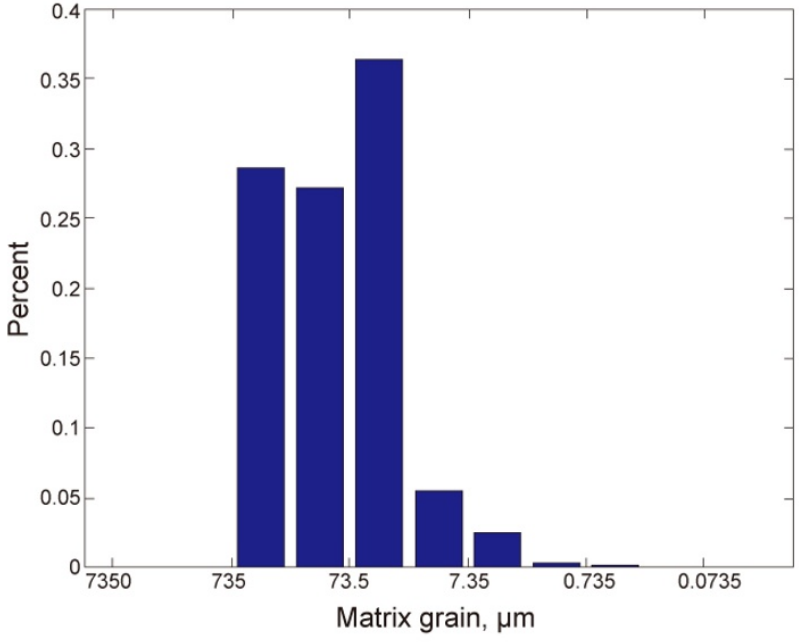

(b)

Fig. 3 Distribution of matrix grains from the CTS images of rock (a) The original CTS image; (b) Radius distribution of matrix grain

Fig. 4 shows the enlarged picture of a small portion of asconstructed porous digital rock. To investigate the electrical properties, the porous medium should be filled with water to form the saturated porous rock model.

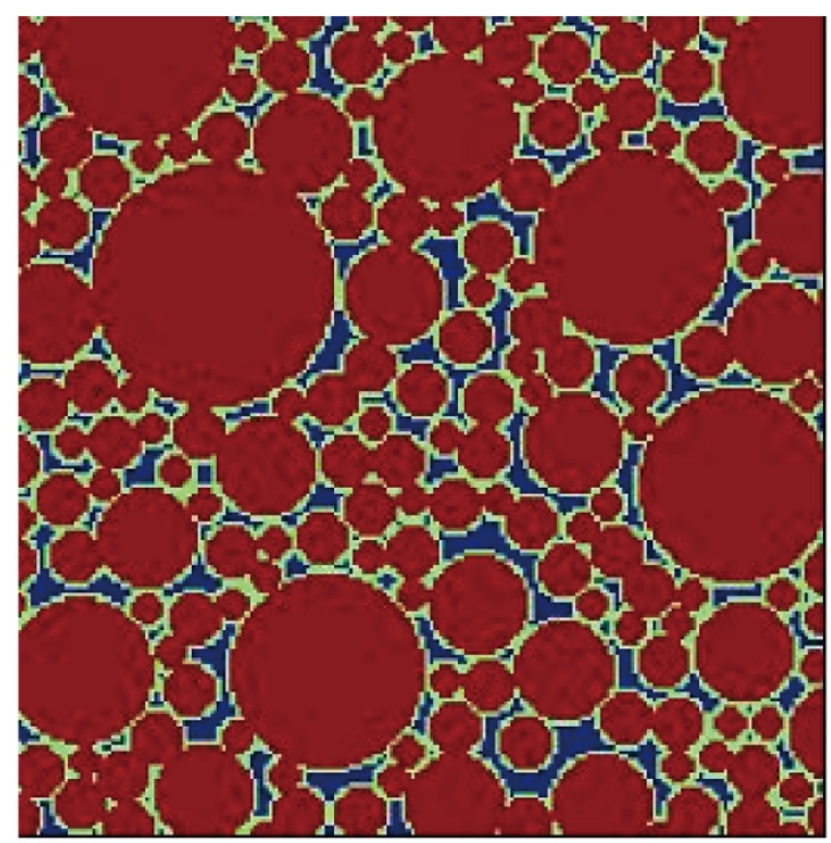

Fig. 4 Section of a constructed digital rock
The electrical transport properties of each phase are dependent on the collision rules of particle evolution (Boon and Noullez, 1989; Küntz et al, 2000). The matrix is of high resistivity in porous medium and the water filling the rock pores is of low resistivity. The definition of porosity and the shapes of matrix grains are as same as that described in literature (Yue et al, 2005). Generally, the complexity of matrix grains can reflect the complexity of the pore space.

\section{Results of numerical simulation}

The LGA method used for simulation of the transport of electrical current is improved by introducing the reflection $(R)$ and transmission $(T)$ coefficients of the border between phases to control the moving direction of particles, different from the conventional LGA method which allows particles moving freely from one phase to another. With the improved LGA method, the choice of current path can be realized automatically by adjusting the reflection coefficient, while keeping the relationship of $R$ and $T$ to meet $T+R=1$.

In the numerical simulations of this paper, the electrical voltages were applied on the rock models in the same way as described by Rothman (1988) and Küntz et al (2000). The upper and lower boundaries of the models are insulated ones as shown in Fig. 5. These can be easily implemented in the LGA method, i.e., non-slip solid boundaries (Li et al, 1994). The left and right boundaries of the model are designed as periodic boundaries to simulate the infinite boundaries in 
the horizontal direction. If $S_{0}(n)$ is taken to represent the state flowing out of the boundary at the nth time step and $S_{i}(n+1)$ the state flowing into the boundary at the $(n+1)$ th time step, then we have $S_{i}(n+1)$ equal to $S_{\mathrm{o}}(n)$. The size of the LGA model used in the simulations is $100 \times 120$ sites. The electrical voltage is set as 0.1 and the initial average density distribution of the particles for the LGA models is 0.85 . For all the simulations, the FHP3, FHP2 and FHP1 collision rules have been used to model the properties of the water filling rock pores, clay content and matrix, respectively.

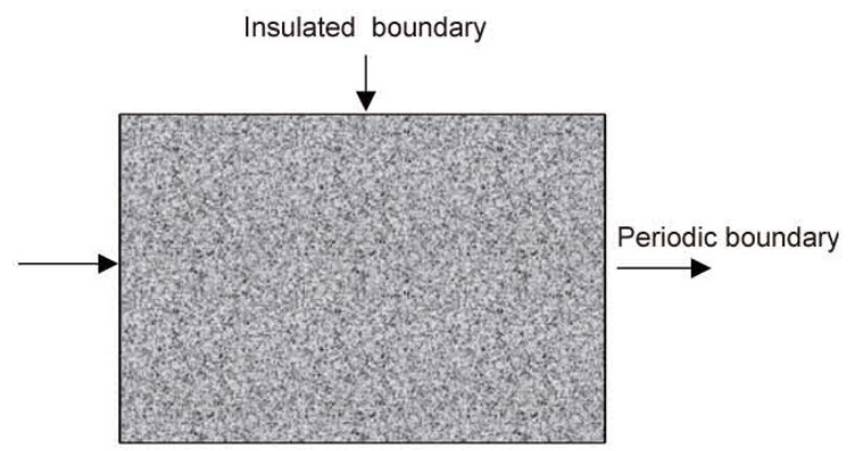

Fig. 5 Sketch of the boundaries of a digital rock model

\subsection{Simulated $\mathrm{F}-\phi$ relationship by LGA}

The simulated $F-\phi$ relationship of the digital rock models by the LGA method is shown in Fig. 6. Through comparing Fig. 6 with Fig.1, it can be seen that the power law relationship of $F-\phi$ with a constant $m$, as described in Archie's equation (1), is obtained by the LGA method with the digital rock model.

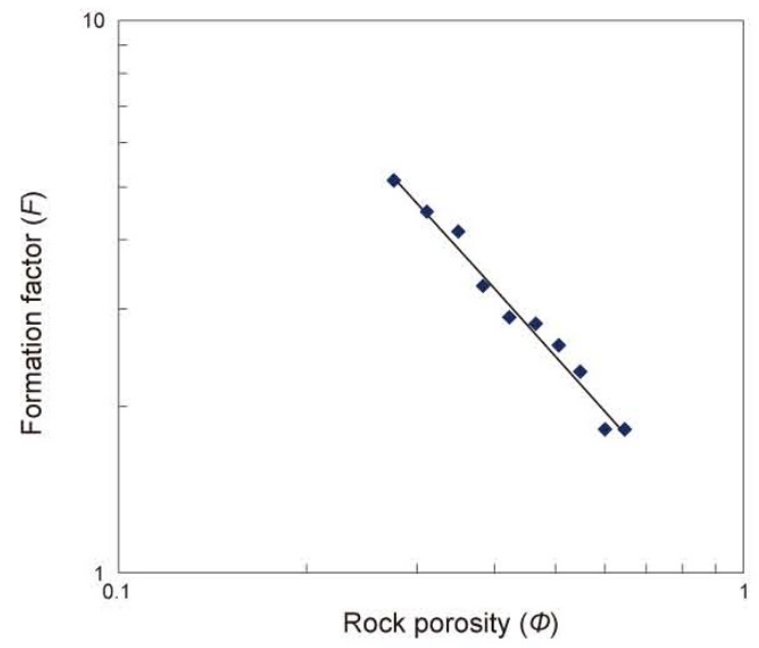

Fig. 6 The $F-\phi$ relationship of digital rocks from LGA simulations

To investigate the influence of pore structure on the electrical transport properties, various geometries of matrix particles such as diamond-shaped, triangle-shaped, point-like and rectangle-shaped particles are used to construct the digital rocks. The complexity of pore structure is determined by the geometries of the matrix particles (Yue et al, 2005) used for the digital rock construction. The relationship between $F$ and $\phi$ obtained by the LGA simulations is similar to each other for different geometries of matrix particles of digital rocks, except for $a$ and $m$.

Study of the data from the LGA simulation shows that a and $\mathrm{m}$ follow the exponential law, as in Fig. 7.

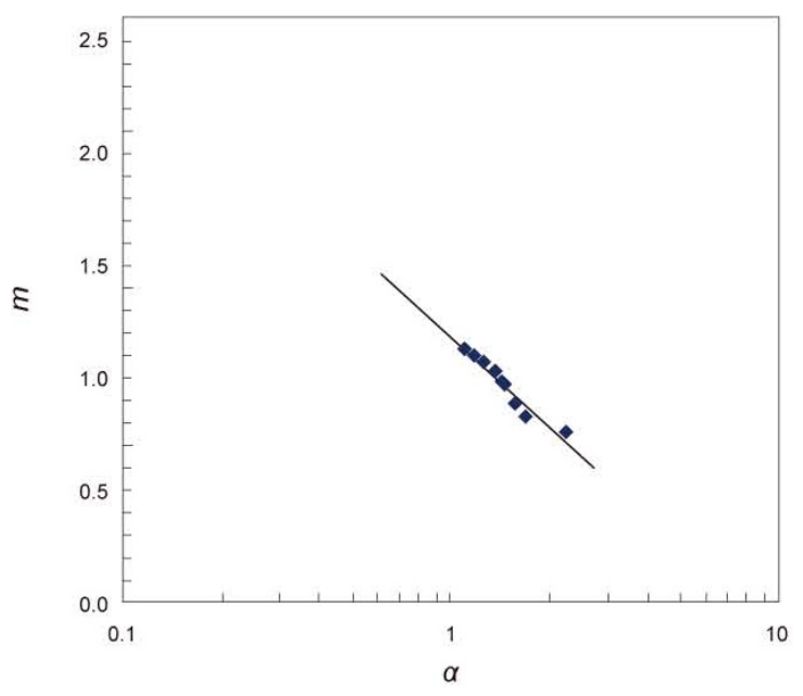

Fig. 7 Relationship of a-m obtained by LGA simulations ( $\mathrm{a}$ is a real constant; $\mathrm{m}$ is the cementation factor)

From Fig. 7, it is clear that the relationship between $a$ and $m$ is linear in a semi log coordinate system. To verify the validity of the results by LGA simulations, the data of $a$ and $m$ obtained by experiments on physical rock is shown in Fig. 8 .

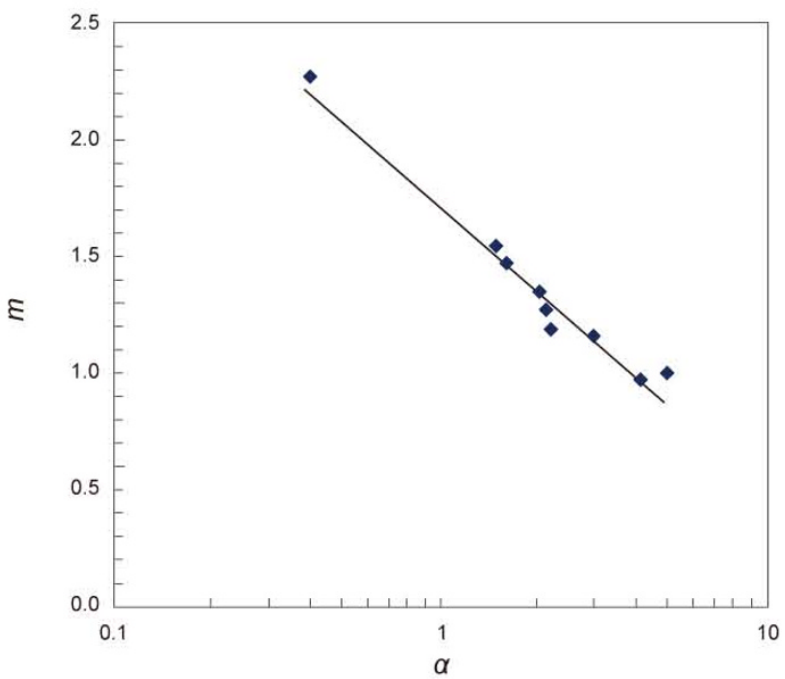

Fig. 8 Relationship of $a-m$ obtained by laboratory experiments using physical rocks ( $a$ is a real constant; $m$ is the cementation factor)

By comparing Fig. 7 and Fig. 8, it is found that the numerical results from the LGA method agrees well with the experimental results from real rock, indicating that the LGA simulation is a reliable method for study of the electrical transport properties, and that the parameters $a$ and $m$ are closely related to the pore structure of porous rocks. 


\subsection{Effects of clay content on the $F-\phi$ relationship}

The clay content has significant effects on the conductivity of a rock. Many researchers believe that Archie's equation only holds true for rock without any clay content (Yong, 1982; Li, 1989; Tao, 1992; Kutay et al, 2007). Based on the hypotheses of parallel conductivity of rock matrix with pores, different models have been developed by taking the effects of clay content into account (Yong and Zhang, 1996). But these models can not be widely used in reservoir evaluation because the micro mechanism of the clay effects is yet not clear from such simplified models.

The applications of LGA to simulate the electrical transport properties of the fluid saturated rocks can reveal the physical mechanisms of the porous rock conductivity at the pore scale. Therefore, the effects of clay content and its distribution on Archie's parameters can be investigated in details by the LGA.

The distribution of clay content in a rock can usually be divided into three types, namely, dispersed, structured and laminated clay distributions. Dispersed clay means that the clay particles are dispersed on the surface of the matrix in the pore space. The existence of dispersed clay will decrease the effective porosity. Structured clay means that the clay particles are an original part of the matrix and have no effects on the effective porosity. Laminated clay means that the clay particles are distributed in the form of a layer replacing the space of matrix and pore in rock. The existence of laminated clay will produce effects on both matrix and effective porosity.

To investigate the effects of clay content by the LGA method using the digital rock model, digital rocks without clay content are constructed first by the method described in section 3, then a specific amount of clay particles, according to the clay content $\left(V_{\mathrm{sh}}\right)$, will be distributed into the digital rock in different distribution types for revealing the effects of clay distribution type on the conductivity of the rock. The clay particles will deposit together with matrix grains to construct the digital rock with structured clay. The random growth method is used for producing the laminar clay, and the random distribution of clay particles is used to produce the dispersed clay according to $V_{\text {sh }}$ in pore space after the deposit of pure matrix grains.

The LGA method is used for numerical study of the effect of dispersed clay on the $F-\phi$ relationship of the digital rock examples and the simulated results are shown in Fig. 9 in log$\log$ scale. The data points of different shapes on the figure represent the simulated results with different Vsh values. Fig. 9 shows that the $F-\phi$ relationship would become nonlinear when the clay content larger than 0.06 . This indicates that the parameter $\mathrm{m}$ (i.e. the slope of the $F$ - $\phi$ relationship) would not be a constant and would decrease with increasing clay content. When the clay content is higher than 0.06 , the $F-\phi$ relationship is obviously not a linear function in loglog scale, as described by Archie's equation. This is the so called non-Archie phenomenon. The reasons for the nonArchie behaviour of $F-\phi$ arelationship lie in the dual effects of porosity reduction and extra conductivity caused by the clay minerals.

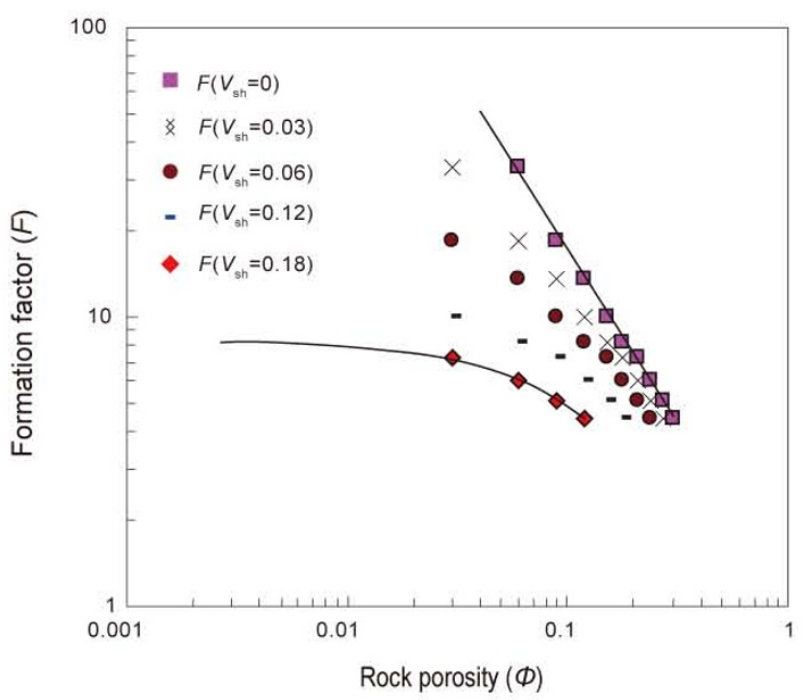

Fig. 9 Effect of dispersed clay on $F-\phi$ relationship

The simulated results for the $F-\phi$ relationship of the digital rocks containing structured clay are shown in Fig. 10. The effects of structured clay on the $F-\phi$ relationship are different from that of dispersed clay, as shown in Fig. 10. The digital rock examples used in this simulation case are the same as the ones for dispersed clay except for the distribution type of structured clay instead of dispersed clay. By comparing Fig. 10 and Fig. 9 , it is found that the changes of the $F-\phi$ relationship with increasing clay content are different for different clay distributions. The reason might be that structured clay, being a part of the rock matrix, has no effect on the pore structure and hence can not reduce the effective porosity. However, structured clay can change the conductive path of the porous rocks due to its extra conductivity. With an increase of structured clay, the additional conductivity makes the current paths simpler. Therefore, the changing rate of the current paths with decreasing porosity in such rocks with structured clay is slower than that in the rocks free of structured clay.

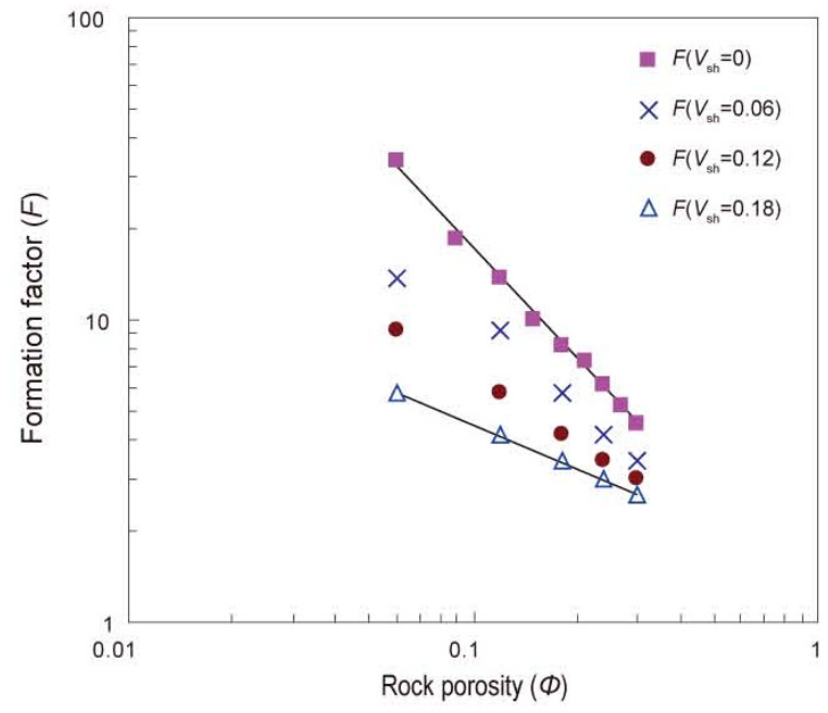

Fig. 10 Effect of structured clay on F- $\phi$ relationship

From Fig. 10, it can also be seen that the $F-\phi$ relationship is always linear in log-log scale for different values of 
structured clay content. The parameter $m$ (the slope of $F-\phi$ relationship) will decrease with increasing clay content. The structured clay may not cause the non-Archie behaviour of $F-\phi$ relationship.

The simulated results for $F-\phi$ relationship of the digital rocks containing laminated clays are presented in Fig. 11. It can be seen that the laminated clay content can cause the reduction of the parameter $m$ and non-Archie behaviour of $F-\phi$ relationship. Moreover, the non-Archie phenomenon caused by the dispersed clay appears only when the porosity is less than 0.1 , while the phenomenon caused by the laminated clay appears when the porosity is less than 0.28 . The reason might be that the laminated clay content can not only reduce the effective porosity of the porous rocks, as the dispersed clay does, but also change the current paths like the structured clay does.

Finally, statistical analysis was done on the simulated results for different clay contents and distribution types to find the dependence of $a$ and $m$ on $V_{\text {sh }}$. These results are given in Fig. 12. In general, the parameter a increases with increasing $V_{\mathrm{sh}}$, while the $\mathrm{m}$ decreases with increasing $V_{\mathrm{sh}}$. The quantitative model for the dependence of a and $\mathrm{m}$ on $V_{\mathrm{sh}}$ is as follows

$$
\begin{array}{lll}
a=1.0591 \cdot e^{1.7896 \cdot V_{\text {sh }}} & R=0.9986 & V_{\mathrm{sh}} \leqslant 0.2 \\
m=1.1446 \cdot e^{-0.0214 \cdot V_{\text {sh }}} & R=0.9874 & V_{\mathrm{sh}} \leqslant 0.2
\end{array}
$$

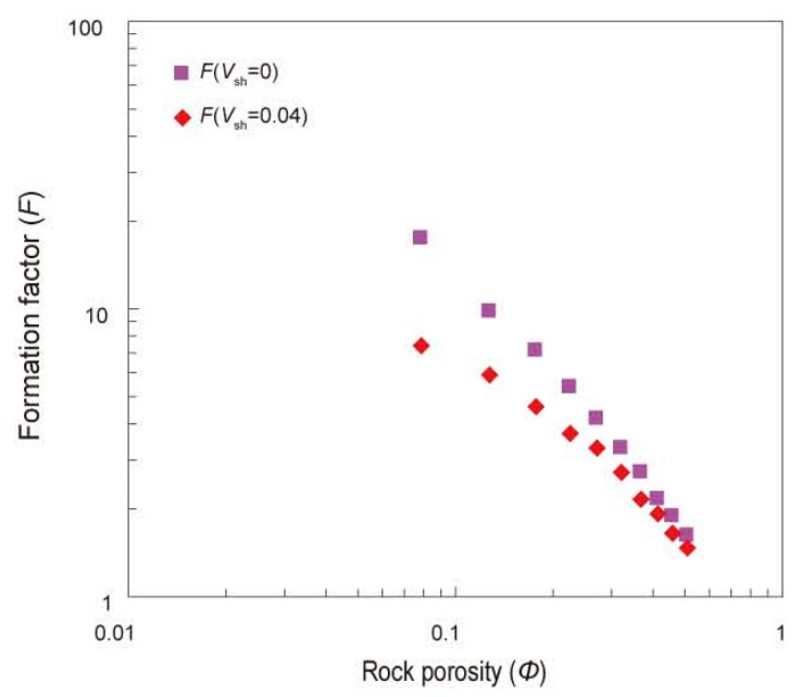

Fig. 11 Effect of laminated clay on $F-\phi$ relationship

It is noted that real rocks have very complex clay distribution and pore structure. The dependence of $a$ and $m$ on $V_{\text {sh }}$ may be determined by experiments using rocks from the actual formations.

Many physical experiments on real rocks show that $m$ depends on $V_{\text {sh }}$ (Saner et al, 1994; Liu et al, 1998). Fig.13 shows the measured results of $m$ and $V_{\text {sh }}$ from experiments with real rocks (Saner et al, 1994).

In Fig.13, it is clear that $m$ decreases with increasing $V_{\text {sh. }}$. The quantitative model for the dependence of $m$ on $V_{\mathrm{sh}}$ is as follows

$$
m=2.0489 \cdot e^{-0.4402 \cdot V_{s h}} \quad R=0.8888
$$

By comparing Fig.12 with Fig.13, it can be found that both the experimental result and the simulated result from the digital rock share the same conclusion, that is, $m$ decreases with increasing $V_{\text {sh }}$.
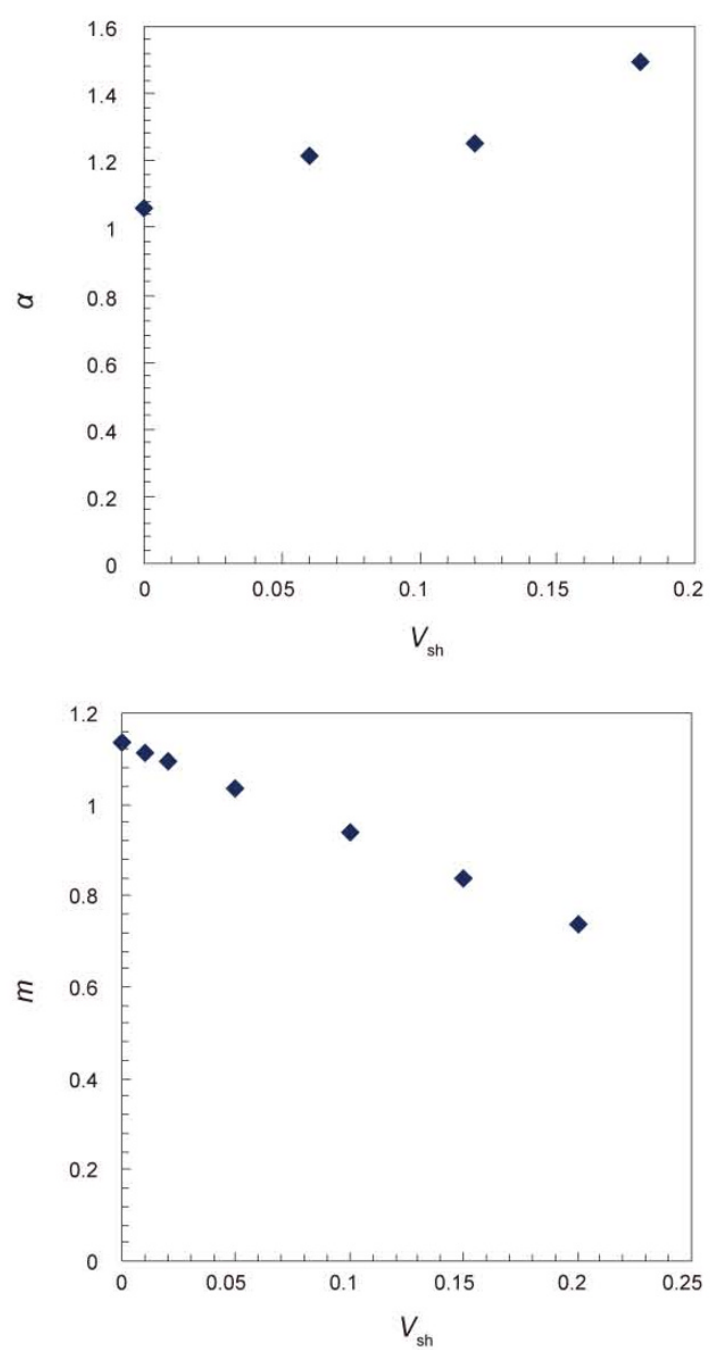

Fig. 12 Relationships between $a, m$ and $V_{\text {sh }}$

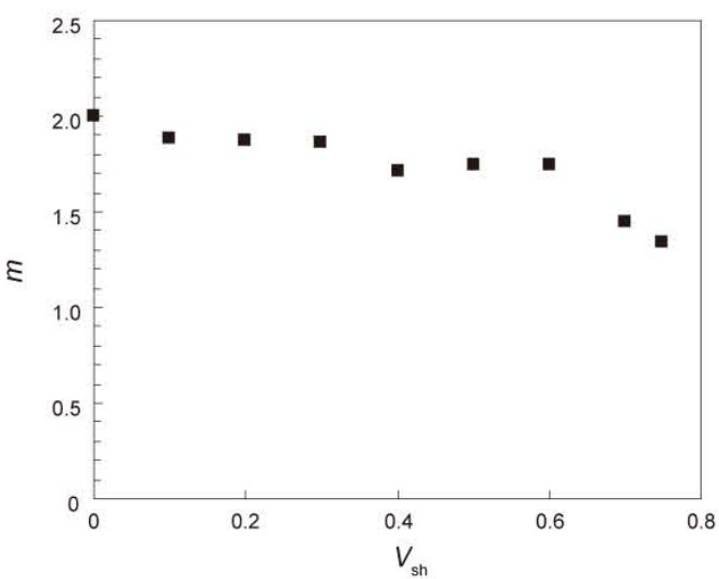

Fig. 13 Relationship between $m$ and $V_{\text {sh }}$ obtained from experiments with real rock (Saner et al, 1994) 
Based on these simulated results, it is found that both dispersed clay and laminated clay can cause non-Archie behaviour of the $F-\phi$ relationship. The non-Archie behaviour of $F-\phi$ relationship caused by laminated clay is more significant than that by dispersed clay. In real world, the distribution of clay contents in porous rocks is complex and could be a mixture of all the three distribution types. The porosity of a real rock is usually less than 0.3 . Therefore, non-Archie behaviour of $F-\phi$ relationship should appear with increasing clay content. Our numerical simulations in this study agree well with the experimental results with real rocks from the literature (Yong, 1982).

\section{Conclusions}

The LGA method has been successfully applied to investigate the effects of clay content and distribution on the $F-\phi$ relationship in this paper. The comparison between the results of the simulations and the laboratory measurements demonstrate the advantages of the LGA over the physical rock experiments in flexibly handling the complex pore structures. The LGA method has proved to be an alternative way for the researchers to reveal the physical meaning of the parameters $a$ and $m$. The numerical results by LGA simulations in this paper have shown that the parameters a and $m$ are closely related to the pore structures of the porous rocks.

Based on these observations, we found that both dispersed clay and laminated clay can cause non-Archie behaviour of the $F-\phi$ relationship. The non-Archie behaviour of the $F-\phi$ relationship caused by laminated clay is more significant than that caused by dispersed clay. In general, the parameter $a$ increases with the increasing of $V_{\text {sh }}$, while the $m$ decreases with the increasing of $V_{\text {sh }}$.

\section{Acknowledgements}

This research was supported by the National Natural Science Foundation of China (projects No. 41074103 and 50404001), and the National Key Fundamental R\&D Project (Grant No. 2007CB209601).

\section{References}

Archie G E. The electrical resistivity log as an aid in determining some reservoir characteristics. Trans. AIME. 1942. 146: 54-61

Boon J P and Noullez A. Lattice gas diffusion and long time correlation, in Monaco R. (ED), Discrete kinematics, lattice gas dynamics and foundations of hydrodynamics. World Scientific, Singapore. 1989. $399-407$

Diederix K M. Anomalous relationships between resistivity index and water saturations in the rotliegend sandstone. 23rd Meeting of the Society of Professional Well Logging Analysts. Corpus Christi, TX. 1982. Paper X.

Frisch U, Hasslacher B and Pomeau Y. Lattice gas automation for the Navier-Stokes equation. Phys. Rev. Lett. 1986. 56: 1505-1507

Hardy J. Time evolution of a two-dimensional model system. J. Math.
Phys. 1973. 14: 1746-1759

Herrick D C and Kennedy W D. Electrical efficiency-A pore geometric theory for interpreting the electrical properties of reservoir rocks. Geophys. 1994. 59(6): 918-927

Hu J X and Li Y M. Modeling research for simulating seismic wave propagation in solid by cellular automata. Chinese J. Geophys. (Acta Geophysica Sinica) 1997. 40(1): 120-125 (in Chinese)

Kutay M E, Aydilek A H and Masad E. Computational and experimental evaluation of hydraulic conductivity anisotropy in hot-mix asphalt. Int. J. of Pavement Engr. 2007. 8(1): 29-43

Küntz M, Mareschal J C and Lavallée P. Numerical estimation of electrical conductivity in saturated porous media with $2 \mathrm{D}$ lattice gas. Geophys. 2000. 65(3): 766-772

Li N. Generalised resistivity-porosity and resistivity-oil saturation relationships. Chinese Journal of Geophysics. 1989. 32(5): 580-591 (in Chinese)

Li X D, Yu J and Li M. Theoretic Research of Reservoir Rock with NonArchie Characteristics. Oil-Gas Field Surface Engineering. 2008. 27(1): 32-33 (in Chinese)

Li Y M and Hu J X. Application of cellular automata approach to the study of seismic wave propagation. Chinese J. Geophys. (Acta Geophysica Sinica) 1995.38(5): 651-661(in Chinese)

Li Y X, Kang L S and Chen Y P. Lattice Gas Automation. Beijing: Tsinghua University Press. 1994

Liu Z F, Liu T Y and Yan J. Analysis of the effects of temperature, pressure, shaliness, wettability and experimental approach on Archie equation. Well Logging Technology. 1998. 22(4): 231-236

Liu J S, Xu Y and Uda B L. Phononic lattice solid with various grid length for modeling seismic waves. Chinese J. Geophys. 1999. 42(4): 536-542 (in Chinese)

Rothman D H. Cellular automation fluids: a model for flow in porous media. Geophys. 1988. 53: 509-518

Saner S, Cagatay M N and Fossail K A. Shaliness effect on the logderived Archie cementation factor: Study of a Saudi Arabian sandstone reservoir. The Log Analyst. 1994. 35(6): 16-27

Tao G. Elastic and transport properties of some sandstones[PhD thesis]. London: Imperial College of Science, Technology \& Medicine, University of London. 1992

Wong P, Koplik J and Tomanic J P. Conductivity and permeability of rocks. Phys. Rev. B. 1984. 30: 6606-6614

Wang $\mathrm{Z} \mathrm{L}$ and $\mathrm{Li} \mathrm{Y} \mathrm{M}$. Parallel algorithm for simulating seismic wave propagation by cellular automata. Chinese J. Geophys. 1999. 42(3): 410-415 (in Chinese)

Worthington P F. Quality assurance of the evaluation of hydrocarbon saturation from resistivity data. SPE Annual Technical Conference and Exhibition, San Antonio, Texas, U.S.A. 2006

Yong S H. Digital process and comprehensive interpretation of well logging curves. Beijing: Petroleum Industrial Press. 1982. 26

Yong S H and Zhang C M. Digital process and comprehensive interpretation of well logging curves. Shandong: University of Petroleum Press. 1996

Yue W Z, Tao G and Zhu K Q. Simulation of electrical transport properties in oil-water saturated porous media with 2-D lattice gas automata. Chinese J. Geophys. 2005. 48(1): 189-195 (in Chinese)

Yue W Z, Tao G, Liu D M, Yang W D. Numerical simulation of nonArchie electrophysical property of saturated rock with lattice Boltzmann method. Petroleum Science. 2009. 6(1): 24-28

(Edited by Zhu Xiuqin) 